\title{
P04-2-5 Poster session
}

\section{Betulinic acid lessens oxidative stress and impairment of aortic contraction induced by lipopolysaccharide in rats}

\author{
Ling-Bo Qian ${ }^{1}$, Yao-Yao Bai ${ }^{1}$, Hui-Ying Zhou ${ }^{1}$, Dong Yan ${ }^{1}$, Wei-Xin Li ${ }^{1}$, Jin-Ting Yang ${ }^{2}$, Xin-Ru Zhou ${ }^{1}$ \\ ${ }^{1}$ Department of Basic Medical Sciences, Hangzhou Medical College, China, ${ }^{2}$ Department of Anesthesiology, the Second \\ Affiliated Hospital, Zhejiang University School of Medicine, China
}

Lipopolysaccharide (LPS) is widely accepted to induce the irreversible vascular hypo-responsiveness in sepsis by oxidative stress. Betulinic acid (BA) has been reported to inhibit oxidative stress and reduce septic lung and kidney injuries. However, whether BA can alleviate the LPS-induced vascular injury remains unclear. To investigate the effect of BA on aortic contraction and oxidative stress in rats exposed to LPS, male Sprague-Dawley rats were pretreated with BA (25 $\mathrm{mg} / \mathrm{kg} / \mathrm{d}$, i.g.) for 3 days and intraperitoneally injected with LPS $(10 \mathrm{mg} / \mathrm{kg})$ at the 4 th day. The contraction of isolated thoracic aorta was determined using the organ bath system. The parameters of inflammatory factor interleukin-6 (IL-6) and oxidative stress were measured. We found that IL-6 was significantly increased in plasma and thoracic aorta from LPS-treated rats and the aortic contraction evoked by phenylephrine, $\mathrm{KCl}$ and $\mathrm{Ca} 2+$ was inhibited by LPS. Malondialdehyde level and inducible nitric oxide synthase (iNOS) activity were all markedly increased, while superoxide dismutase (SOD) activity was significantly decreased in the thoracic aorta from LPS-treated rats. All these alterations were reversed by pretreatment with BA. These findings indicate that BA improves aortic contraction in LPS-treated rats, which may associate with activating SOD and inhibiting oxidative stress and iNOS activity.

Xin-Ru Zhou is the corresponding author.

Acknowledgments

This work was supported by National Natural Science Foundation of China (81772035 and 81600203 ), Foundation from Science and Technology Department of Zhejiang Province (2016C37133), Chinese Medicine Research Program of Zhejiang Province (2016ZA045), and Natural Science Foundation of Hangzhou Medical College (2016XZA04). 